\title{
Progress on The Path to A Licensed Vaccine Against Plasmodium Falciparum Malaria
}

\author{
Andrew W. Taylor-Robinson \\ School of Medical \& Applied Sciences, Central Queensland University, Rockhampton, QLD 4702, Australia
}

\section{CorResPonding AUTHOR :}

Andrew W. Taylor-Robinson School of Medical \& Applied Sciences Central Queensland University Rockhampton QLD 4702 Australia

E-mail: a.taylor-robinson@cqu.edu.au

Received: 18.06.2014

Accepted: 19.08 .2014

Published: 21.08.2014

(c) This article is distributed under the terms of the Creative Commons Attribution License, which permits unrestricted use and redistribution provided that the original author and sourceare credited.

Competing interests: The author has declared that no competing interests exist.

\section{Access This Article}

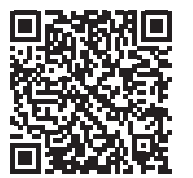

ABstract

Infection with Plasmodium falciparum, the cause of the most severe form of malaria, kills at least one million people each year, mainly children, in addition to significant debilitation in further hundreds of millions. Consequently, this disease has a profound socioeconomic impact in endemic countries and hence it has been a focus of global health initiatives for many years. Increased investment in existing control measures, such as insecticide-impregnated bed nets, has been matched by renewed efforts to develop an efficacious vaccine. Sequencing of the genome of $P$. falciparum has improved knowledge of the malaria parasite's complex lifecycle, which, combined with a greater understanding of the human immune response to infection, has spawned several novel candidate vaccines over the last two decades. Most notable among these is RTS,S which has shown much promise during a long development process, recently becoming the first candidate vaccine against human malaria to progress into phase 3 clinical trials. There is optimism, therefore, that in the foreseeable future RTS,S may become the first ever licensed vaccine against a parasitic disease in humans. However, this is tempered by unresolved issues surrounding the lack of understanding of its mechanism of protection and doubt cast over its long-term therapeutic potential. While the future of RTS,S as a commercially available product is not certain, it should contribute to the continuing campaign against malaria, if only as a prelude to a more refined second generation vaccine.

Keywords: Malaria, Plasmodium falciparum, Immunity, Vaccine, RTS,S.

\section{INTRODUCTION}

Malaria in humans is caused by five species of protozoan parasite from the genus Plasmodium which is transmitted by Anopheles mosquitoes. ${ }^{[1,2]}$ The disease is currently endemic on every continent, save for Antarctica, but is confined to mainly tropical regions. ${ }^{[3,4]}$ Of the estimated global population of 7.1 billion people, ${ }^{[5]} 3.4-3.7$ billion are at risk of contracting malaria. ${ }^{[3,6]}$ It is routinely claimed that there are $300-500$

Cite as: Taylor-Robinson AW. Progress on The Path to A Licensed Vaccine Against Plasmodium falciparum Malaria. J Immunol Immunotech. 2014:1 (1)-1-14. 
million clinical cases worldwide annually. ${ }^{[7]}$ The 1-3 million deaths that result equate to a death every 10-30 seconds. ${ }^{[8,9]} 90 \%$ of deaths are due to P. falciparum and occur principally in young children in sub-Saharan Africa. Pregnancy-associated malaria is the cause of 75,000-200,000 infant and maternal deaths a year. [10] The distribution of malaria is mainly among socioeconomically lower developed countries, further perpetuating their cycle of poverty. It is calculated to cost these endemic nations approximately US $\$ 12$ billion a year. ${ }^{[3,11]}$ Annual losses of $1.3 \%$ in economic growth may be attributed in part to lost person hours and therefore to reduced productivity. ${ }^{[12]}$ Furthermore, children who miss school suffer from deficient education which ultimately affects their future employment prospects.

These statistics bear witness to a human public health catastrophe. An efficacious and cost-effective vaccine against $P$. falciparum, or the debilitating and frequently life-threatening effects infection with this parasite causes, is considered a holy grail of modern molecular medicine. ${ }^{[13]}$ Clinical studies in humans have consistently related immune responses to antigens expressed during pre-erythrocytic and erythrocytic stages of the malaria life cycle with resistance to infection or disease, providing a powerful rationale for development of anti-malaria vaccines. By dissecting the mechanism(s) of immunity to antigens expressed by liver and blood stage parasites, we can best evaluate in different delivery systems epitopes associated with protection as components of a focused and coordinated multi-antigen malaria vaccine strategy. This review examines the hurdles to successful vaccine design presented by the parasite and highlights the major issues which are required to be addressed surrounding potentiation of a putatively protective immune response. The prospects for strategies currently in development, notably RTS,S, are discussed.

\section{Parasite lifecycle and Disease PATHOPHYSIOLOGY}

Malaria has a multi-stage lifecycle which is broadly similar for all Plasmodium species that infect humans. Differences in the requirements for host cell invasion of each species lead to different aetiologies and therefore different clinical presentations.A person becomes infected when they are inoculated with Plasmodium sporozoites harboured in the saliva of a female Anopheles mosquito during the course of it taking a blood meal. Once the sporozoites enter the peripheral blood they home rapidly to the liver, invade hepatocytes and undergo asexual reproduction (hepatic schizogony) to produce intracellular liver schizonts. ${ }^{[14-16]}$ In the case of $P$. vivax and $P$. ovale but not $P$. falciparum, a dormant form of the parasite, the hypnozoite, may also occur. ${ }^{[14,17]}$ Hypnozoites also grow within hepatocytes but remain quiescent and are responsible for persistence of infection when malaria relapses after the initial infection has been cleared. ${ }^{[18]}$ This pre-erythrocytic stage of the lifecycle has been the target of the majority of vaccine research to date. ${ }^{[15]}$

When schizont-infected hepatocytes rupture they release thousands of merozoites into the blood which invade erythrocytes and undergo another asexual reproductive cycle (erythrocytic schizogony). ${ }^{[14,16]}$ Merozoites feed on haemoglobin and develop into trophozoites, then schizonts. When an erythrocyte ruptures, yet more merozoites are released, vastly increasing the parasitaemia, the parasite load in the blood; this is responsible for the manifestations of acute uncomplicated malaria. ${ }^{[14,15]}$ The classic clinical signs are febrile paroxysms, which are rolling fevers consisting of three stages - the cold stage (cold, rigors), hot stage (spiking temperature) and sweating stage. ${ }^{[14]}$ In the case of $P$. falciparum, this cyclic fever runs every two days, separated by a short period where the host feels slightly better before another cycle begins. Paroxysms are cytokine-induced and relate directly to erythrocyte rupture. ${ }^{[17]}$ Fever is frequently accompanied by other manifestations such as headache, myalgia, malaise, confusion, anxiety and hepatosplenomegaly due to engorgement with infected erythrocytes. ${ }^{[14,16]}$

Figure 1 illustrates the lifecycle for $P$. falciparum and shows the three stages that form targets - pre-erythrocytic vaccines, blood stage vaccines and transmission blocking vaccines (block sporogenic cycle). There

Cite as: Taylor-Robinson AW. Progress on The Path to A Licensed Vaccine Against Plasmodium falciparum Malaria. J Immunol Immunotech. 2014:1 (1)-1-14. 
is temporal expression of a great number of stage-specific antigens for which experimental vaccines are designed to target. Severe P. falciparum infection (complicated malaria) can manifest in a number of ways including cerebral malaria (cytoadherence of infected erythrocytes in the brain with neurological sequelae), severe anaemia, renal disease and pulmonary oedema, and these complications collectively cause most deaths. ${ }^{[16]}$ Therefore, a pre-erythrocytic vaccine strategy aims to eliminate infection prior to the release of parasites into the blood with which morbidity and mortality is associated.

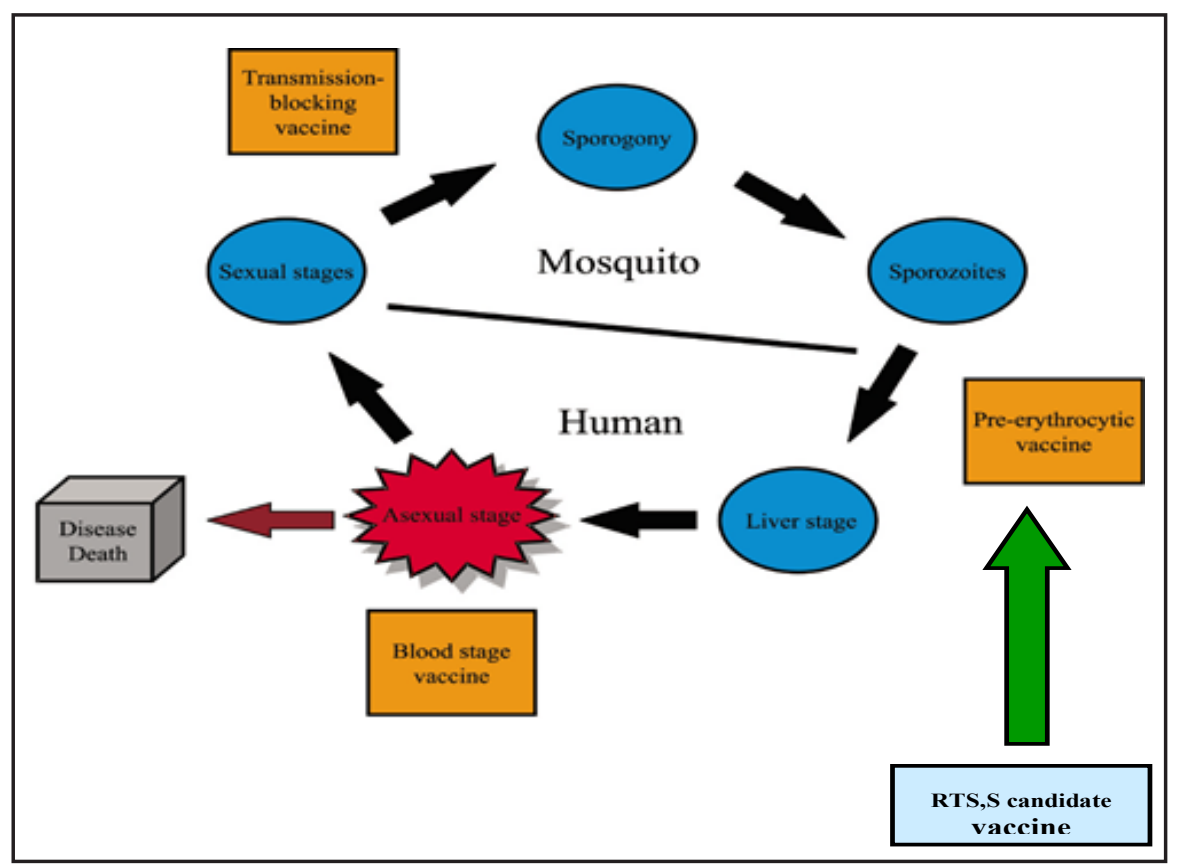

Figure. 01 Schematic life cycle of Plasmodium falciparum, indicating where vaccination may be expected to intervene. A pre-erythrocytic candidate, such as the RTS,S vaccine, targets the parasite before asexual stages are released into the blood, causing the pathology of the disease.

\section{Global StRATEgies for malaria CONTROL - PAst AND PRESENT}

Large-scale attempts to control malaria have a turbulent history, successful in certain parts of the world but faltering badly in many others, and best practice continues to be neglected. ${ }^{[19]}$ There are ample ways to prevent or lessen the risk of contracting infection, and elimination and ultimately eradication are considered theoretically possible with recommended preventative measures. Current strategies include: prevention through mosquito vector control and use of long-lasting insecticide-impregnated bednets and, in some settings, indoor residual spraying with insecticides; seasonal malaria chemoprevention as appropriate; intermittent preventive treatment for infants and during pregnancy; prompt diagnostic testing; and treatment of confirmed cases with effective anti-malarial drugs. ${ }^{[20]}$ These low technology tools have reduced dramatically the disease burden of $P$. falciparum in some regions of Africa and will continue regardless of any future deployment of a first-generation malaria vaccine. However, malarious areas are concentrated mainly in developing countries where the economic resources to implement effective and sustained nationwide coverage are often lacking. Furthermore, poor education, living conditions and malnourishment, for example, exacerbate the situation. There are also problems with drug resistance within the parasite and insecticide resistance within the mosquito due to selection pressures imposed by their overuse and misuse. ${ }^{[21]}$

Cite as: Taylor-Robinson AW. Progress on The Path to A Licensed Vaccine Against Plasmodium falciparum Malaria. J Immunol Immunotech. 2014:1 (1)-1-14. 
THE PRESSING NEED FOR A MALARIA VACCINE

Poor implementation and not the quality of cost-effective control strategies per se is ultimately responsible for our failure to eradicate malaria. Consequently, although currently not available, a licenced vaccine may offer the only hope of eradication; this worldwide public health objective was accomplished for smallpox, which sets a precedent for all other infectious diseases, including malaria. Rational design of a malaria vaccine is based on understanding natural immunity, then artificially potentiating protective responses to immunogenic antigens. Once a candidate is identified, it starts the lengthy process of formulation, enhancement and eventually testing in clinical trials.

In the last decade, a resurgence of public awareness of the 'malaria problem' called for an increased effort in the use of control measures. This prompted The Bill and Melinda Gates Foundation (BAMGF) in 2007 to announce that eradication should be attempted again. ${ }^{[19,22]}$ Rapid support from the World Health Organization (WHO) led to the formation of the Malaria Elimination Group, which is now working toward this. In addition, in 2004 the Program for Appropriate Technology in Health (PATH) led the formation of the Malaria Control and Evaluation Partnership in Africa. ${ }^{[23]}$ This works with the continent's governments and the Roll Back Malaria partnership to deliver control and prevention interventions.

Through a consortium of the world's leading health organizations including the WHO, BAMGF and the Wellcome Trust, in 2006 the Malaria Vaccine Technology Roadmap was created. This outlined a very ambitious new goal to produce a vaccine by 2025 that has a protective efficacy of over $80 \%$ against clinical disease caused by $P$. falciparum and lasts for at least four years. ${ }^{[23]}$ An important milestone was outlined that by 2015 the vaccine should be $50 \%$ effective against severe disease and death and should last for over a year.

IMMUNITY TO MALARIA UNDERPINS VACCINE RATIONALE

\section{Correlation with exposure}

Immunity of humans to P. falciparum develops slowly in endemic areas and is attributed to the parasite's low immunogenic nature and high antigenic polymorphism which makes immunity strain-specific. [24,25] Consequently, this necessitates cumulative exposure to many variants of a particular species which usually requires the first twenty years of life to develop fully. ${ }^{[24,26]}$ Infections are more severe in the young; hence the majority of malaria-related deaths are in children. ${ }^{[4,27]}$ In endemic areas, $P$. falciparum infection in children under the age of 5 years can result in severe disease and death, accounting for nearly $25 \%$ of childhood mortality in Africa. Cerebral malaria and respiratory distress, the most severe manifestations of disease, share many of the features of uncontrolled inflammation or sepsis. These may be studied in detail using well-defined experimental models. ${ }^{[28]}$ In addition, pregnant women show increased susceptibility, in part because the placenta provides a new adult tissue to which pregnancy-associated parasite variants that express the VAR2CSA antigen adhere via specific binding to chondroitin sulphate A on the surface of syncytiotrophoblast cells. ${ }^{[29]}$ Parasite accumulation, accompanied by inflammation, disrupts the cytokine balance of pregnancy with the potential to cause placental damage, compromise foetal growth and trigger preterm delivery. Immunity to placental malaria parasites develops in a gravidity-dependent manner which prevents unfavourable pregnancy outcomes in multigravid women. ${ }^{[29,30]}$

In general, repeated exposure elicits non-sterile immunity whereby individuals have a low level parasitaemia but with little or no symptoms of disease; this is sometimes referred to as stable malaria. ${ }^{[31,32]}$ Although the adult immune system can control infections, asymptomatic individuals are still reservoirs of infection; furthermore, immunity wanes rapidly unless an individual is continually re-infected, making immunity imperfect.

Cite as: Taylor-Robinson AW. Progress on The Path to A Licensed Vaccine Against Plasmodium falciparum Malaria. J Immunol Immunotech. 2014:1 (1)-1-14. 
[31-33] Individuals with waning immunity or irregular infection therefore have unstable malaria. ${ }^{[4]}$ These reasons further highlight the need for an effective vaccine which would induce sterile immunity, i.e. a complete absence of the parasite from a would-be host, without requiring years of exposure and debilitating illness.

\section{Identifying the knowledge deficit}

The fact that inhabitants of regions endemic for $P$. falciparum acquire immunity to 'mild' or 'uncomplicated' malaria only after many years' exposure to numerous bites from infectious mosquitoes raises several key questions regarding the nature of a protective immune response. In order to critically inform effective vaccine design, the fundamental question of what it is that constitutes immunity to P. falciparum needs to be resolved. Specifically, insight is required on several issues:

- Which of the over 5400 gene products encoded by P. falciparum are targets of innate and adaptive immunity?

- Do any of these products interfere with acquisition of immunity, and how does this occur?

- Why does acquisition of immunity take so long to develop?

- How is generation of protection influenced by the frequency or persistence of infection?

- How long does this immunity last, and in what form, in the absence of continuing parasite exposure?

- Are the same or related mechanismsinvolved in protection from severe and mild malaria?

- How do multigravid women develop parasite-specific immunity which prevents unfavourable pregnancy outcomes?

- How are P. falciparum infection and acquisition of immunity influenced by co-infection with other pathogens, e.g. HIV/AIDS, tuberculosis or schistosomiasis, superinfection with an antigenically variant isolate of $P$. falciparum or the individual's own microbiota?

- If a vaccine is partially effective, is there a difference between the protective immune response elicited and that induced by natural $P$. falciparum infection, and if so, is this significant?

- Is there a particular immune response induced by a candidate malaria vaccine which provides a reliable correlate of protection?

- Is there an early molecular marker induced by vaccination which accurately predicts the subsequent quality, magnitude and longevity of a protective effector and memory response?

Answers to all these questions will inform the optimal design of a malaria vaccine. An ideal vaccine would have a high efficacy in all ages of recipient, be safe to administer, safe inside the host and not have any serious side-effects. ${ }^{[34]}$ It should also reproduce, or better still, improve upon naturally acquired immunity and be long-term without necessary boosters or interference from other vaccines. ${ }^{[35]}$ Finally, it should be inexpensive to manufacture and purchase. Unfortunately, malaria vaccines are unlikely to fulfil some of this 'wish list' of properties. Instead, it is more probable that they will be moderately effective, confer short-term immunity and be difficult and expensive to make. ${ }^{[34-36]}$

\section{Malaria vaccines - Current State}

Due to the shortfall in effectiveness of other malaria control measures, increased attention on vaccines led to the formation in 1999 of the PATH Malaria Vaccine Initiative (MVI), a non-government organization (NGO) based in Washington DC, USA, which facilitates accelerated testing of malaria vaccines. ${ }^{\left[{ }^{[3]}\right]}$ There are currently around 63 documented malaria vaccine candidates, including 41 in preclinical and clinical trials. ${ }^{[38]}$ Four vaccines are currently supported by MVI in clinical development (Figure 2).

Candidate vaccines aimed at the pre-erythrocytic stage target either sporozoites or the intrahepatic para-

Cite as: Taylor-Robinson AW. Progress on The Path to A Licensed Vaccine Against Plasmodium falciparum Malaria. J Immunol Immunotech. 2014:1 (1)-1-14. 
site. ${ }^{[39]}$ Designed to induce sterile immunity against antigens found on inoculated sporozoites or expressed by schizont-infected hepatocytes, their effectiveness is based on preventing hepatocyte invasion and so the development of merozoites, which would otherwise invade erythrocytes. ${ }^{[16]}$ With a relatively large period within which to operate (seven days for P. falciparum) and a small number of infectious organisms with which to deal, this a promising stage to target. ${ }^{[40]}$ It is a clinically silent stage and so an effective vaccine would prevent clinical symptoms developing plus halt transmission.

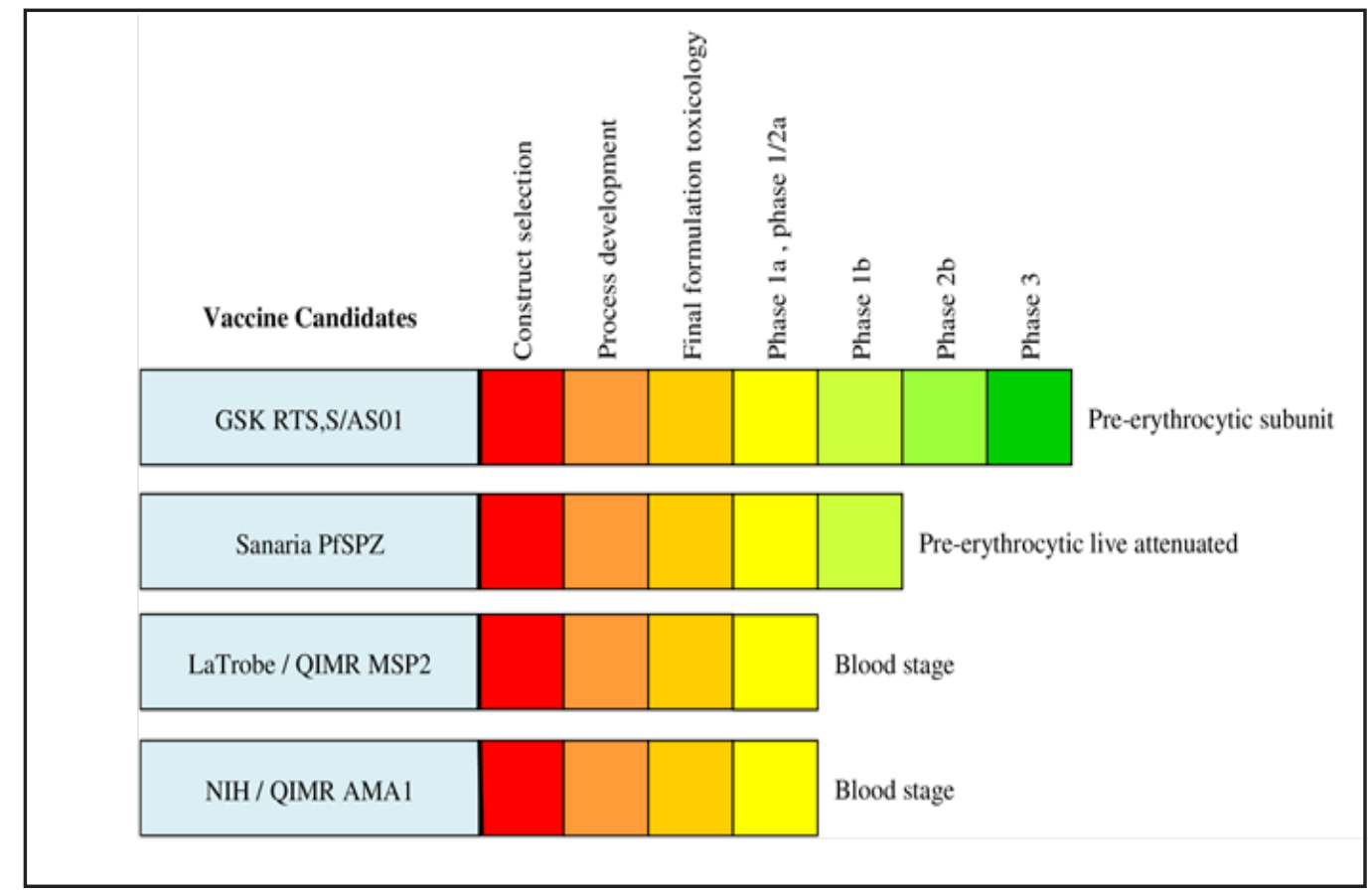

Figure. 02 Diagram to show the relatively advanced progress of RTS,S in comparison to development of

\section{other leading malaria candidate vaccines.}

In 2001, MVI reached an understanding with GlaxoSmithKline (GSK) todevelop a promising candidate vaccine against $P$. falciparum called RTS,S, which was originally created in 1987 in collaboration with the Walter Reed Army Institute of Research. ${ }^{[41]}$ Based on the circumsporozoite protein (CSP) that coats the surface of $P$. falciparum sporozoites and which has been linked strongly to effective human immune responses, ${ }^{[42]}$ RTS,S has been shown to elicit sterile immunity in malaria-naive and semi-immune individuals. By 2006 , an additional \$207.6 million had been invested which has helped advance trials of the RTS,S/AS01 formulation, ${ }^{[41]}$ which in March 2009 became the first vaccine to advance as far as phase 3 clinical development. [9] (Figure 2).

\section{DeVELOPMENT OF RTS,S}

\section{Molecular structure}

RTS,S is a hybrid molecule consisting of two recombinant segments (RTS and S) co-expressed in a Saccharomyces cerevisiae yeast cell. ${ }^{[43]}$ RTS comprises three distinct portions, the first of which is a single polypeptide chain corresponding to a large region of the highly conserved tandem repeat $(R)$ tetra-peptide sequence from CSP; each repeat tetra-peptide is called NANP. ${ }^{[4,45]}$ There are 19 copies of NANP and it is named for its amino acid sequence.

Cite as: Taylor-Robinson AW. Progress on The Path to A Licensed Vaccine Against Plasmodium falciparum Malaria. J Immunol Immunotech. 2014:1 (1)-1-14. 
The second portion is the T lymphocyte epitope-containing $(T)$ flanking region on the C-terminus; this region is made up of highly conserved amino acid sequences separated by immunodominant effector CD4 ${ }^{+}$ and $C D 8^{+}$epitopes known as Th2R and Th3R. ${ }^{[42,44]}$ The ' $R$ ' and ' $T$ ' components correspond to amino acids 207-395 on CSP of the 3D7 standard laboratory strain of $P$. falciparum. ${ }^{[42,44,45]}$ Figure 3 is a simplified representation of the 'RT' portion. This RT peptide chain is then fused to the $\mathrm{N}$-terminal of the Hepatitis $\mathrm{B}$ surface (S) antigen (HBsAg), hence RTS. ${ }^{[43]}$ The second segment (S) is also a polypeptide chain and is 226 amino acids in length; it is an unfused $S$ antigen that corresponds to HBsAg. ${ }^{[42]}$ These recombinant proteins self-assemble into virus-like composite particulates, allowing for increased uptake and expression by antigen-presenting cells. ${ }^{[43,44]}$

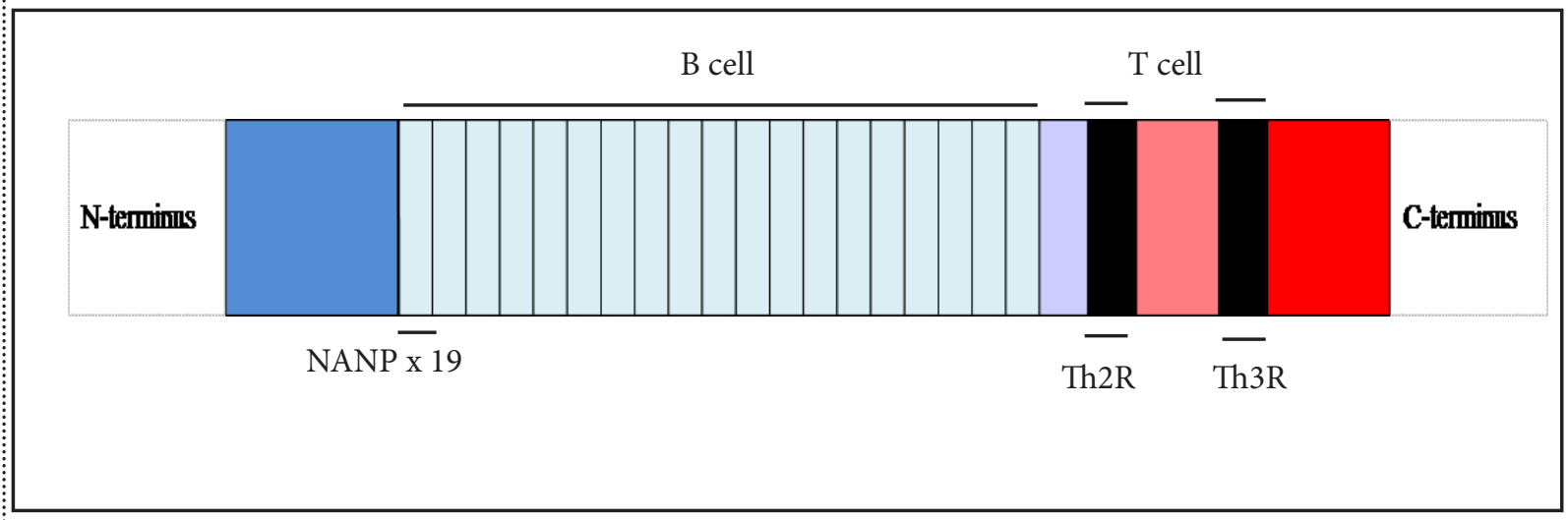

Figure. 03 Schematic diagram of the 'RT' section of the RTS, S construct.

HBsAg is used for its high cell-mediated and humoral immunogenicity; this property is effectively a secondary boost to the immune response which then has a primary effect on a sporozoite challenge. ${ }^{[46]}$ Furthermore, using HBsAg stimulates immunity to Hepatitis $B$ as well, making this a potentially bivalent vaccine. ${ }^{[10,41]}$

\section{Immune response that underpins RTS,S design}

The exact processes involved in naturally acquired pre-erythrocytic stage immunity remain poorly defined. ${ }^{[47]}$ Nevertheless, it is known that the first line of defence is antibody-producing B cells that target newly inoculated sporozoites. ${ }^{[48]}$ This humoral immune response is a consequence of $B$ cell activation after recognition of the repeat tetra-peptide. The antibodies involved are predominately polyclonally produced non-specific immunoglobulin ( $\mathrm{Ig}) \mathrm{M}$ and $\mathrm{IgG}$ but a small proportion, around $5 \%$, are species- or stage-specific and can interact with more of the parasite antigens. ${ }^{[49]}$ The time frame for the immune system to act on the sporozoites before they infect hepatocytes is short, and they are rarely cleared in this time. Once the hepatocytes become infected the cellular immune response takes over, which involves $\mathrm{CD} 4^{+}$and $\mathrm{CD} 8^{+} \mathrm{T}$ cells.

$\mathrm{CD} 8^{+} \mathrm{T}$ cells are implicated in the response to intrahepatocyte infection through a number of mechanisms. [48,50] One of these uses cytolytic activity and works via the recognition of infected cells displaying parasite fragments in association with major histocompatibility complex class I. $^{[1]}$ Recognition induces the secretion of cytotoxins and pore-forming perforins, which ultimately cause cell death, thus killing the parasite. This ability gives rise to its alternate name of cytotoxic $\mathrm{T}$ cells; however, $\mathrm{CD} 8^{+} \mathrm{T}$ cells can also produce cytokines, especially interferon gamma $(\mathrm{IFN}-\gamma) \cdot{ }^{[51]}$

IFN- $\gamma$ has both immunostimulatory and immunomodulatory properties and its production is known to play a more significant role than cytolytic activity toward infected hepatocytes. ${ }^{[48,50]}$ One of its mechanisms of action is based on the induction of the nitric oxide pathway, which is thought to be largely responsible for destroying

Cite as: Taylor-Robinson AW. Progress on The Path to A Licensed Vaccine Against Plasmodium falciparum Malaria. J Immunol Immunotech. $2014: 1$ (1)-1-14. 
infected hepatocytes. ${ }^{[50,52]}$ Although $\mathrm{CD}^{+} \mathrm{T}$ cells are important, the quantities found in sporozoite-infected individuals are not high enough to account for the IFN- $\gamma$ levels found. ${ }^{[53]}$ Other IFN- $\gamma$-producing cells are therefore principally responsible for its production; $\mathrm{CD} 4^{+} \mathrm{T}$ cells, natural killer cells, natural killer $\mathrm{T}$ cells and $\gamma \delta \mathrm{T}$ cells (a rare form of $\mathrm{T}$ lymphocyte). ${ }^{[47,48]} \mathrm{In}$ fact, $\mathrm{CD} 4^{+} \mathrm{T}$ cells produce the highest levels of IFN- $\gamma$ and murine studies have shown $\mathrm{CD}^{+}{ }^{+} \mathrm{T}$ cells (mainly $\mathrm{T}$ helper 1 ; Th1) to be essential to controlling parasitaemia. ${ }^{[43]}$ Furthermore, depletion of $\mathrm{CD}^{+} \mathrm{T}$ cells still preserves IFN- $\gamma$ levels but depletion of $\mathrm{CD}^{+} \mathrm{T}$ cells does not. ${ }^{[4]}$ These observations were made during a study involving RTS,S and help to explain the reasoning behind the design of the construct.

\section{Mechanism of action of RTS,S}

RTS,S works in two ways to induce an immune response against a malaria infection (Figure 4). Firstly, it induces antibody-producing B cells by mimicking the repeat tetra-peptide of CSP. ${ }^{[40,54]}$ This process and

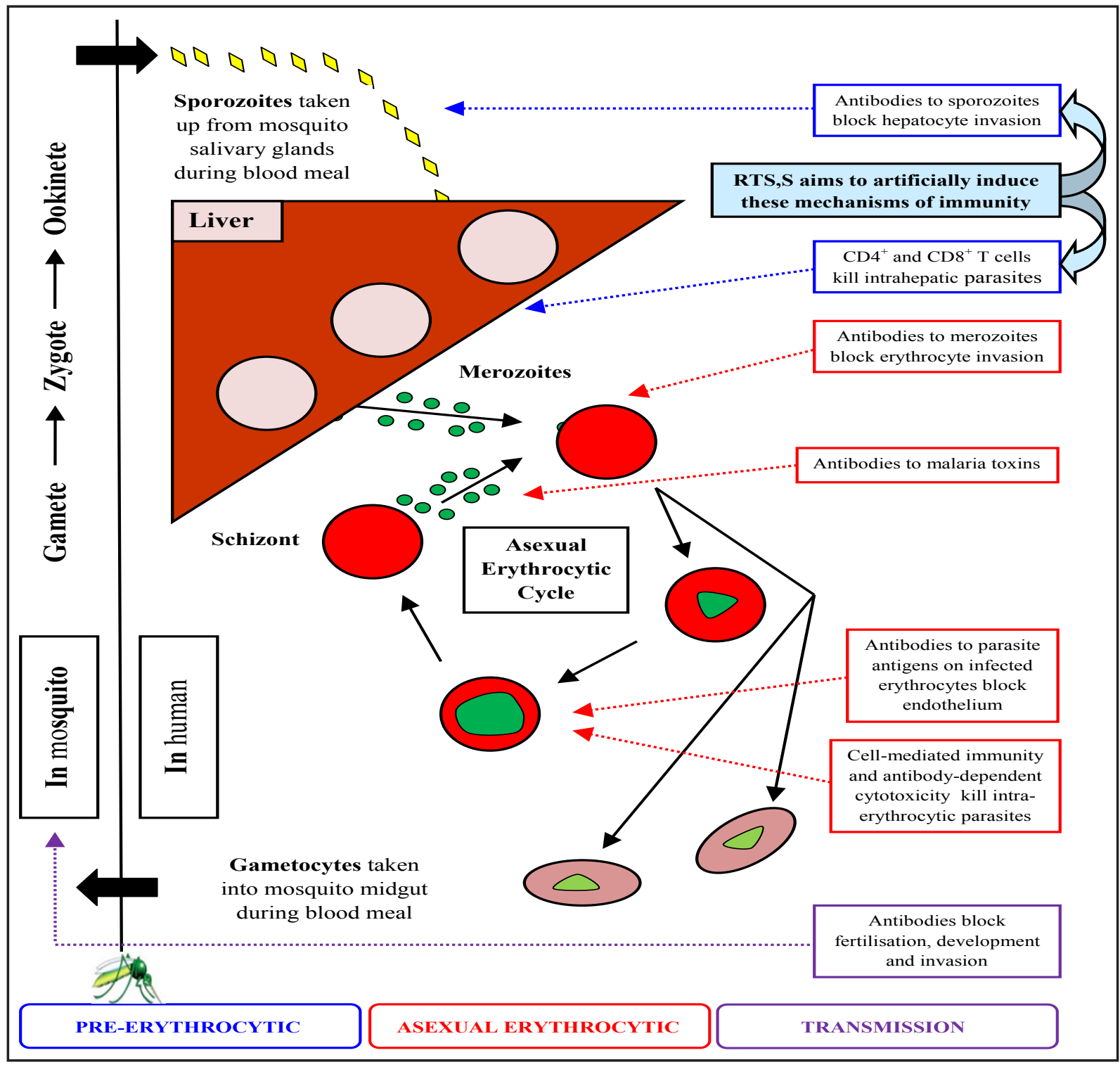

Figure. 04 Schematic diagram showing the mechanisms of immunity against different life cycle stages of P. falciparum, highlighting those against the pre-erythrocytic parasite that RTS,S aims to induce.

Cite as: Taylor-Robinson AW. Progress on The Path to A Licensed Vaccine Against Plasmodium falciparum Malaria. J Immunol Immunotech. $2014: 1$ (1)-1-14. 
the subsequent B cell proliferation and differentiation blocks sporozoite entry into hepatocytes. This induction acts effectively as an immune booster because, as discussed, the immune system alone would rarely clear all the sporozoites. The second approach is via the induction of high levels of IFN- $\gamma$ by stimulating IFN- $\gamma$-producing Th1 CD4 ${ }^{+} \mathrm{T}$ cells that recognise the specific $\mathrm{T}$ cell epitopes on the RTS construct. ${ }^{[48,50]}$ It also induces $\mathrm{CD}^{+}{ }^{+}$cells but to a lesser extent. ${ }^{\left[{ }^{[5]}\right.}$ As IFN- $\gamma$ is involved in inhibiting parasitic development within hepatocytes its production further improves the vaccine's effectiveness. RTS,S only targets conserved antigens to avoid exerting a selection pressure which could lead to parasite resistance.

Sporozoites are not particularly immunogenic and, due to its close relation to CSP, the same can be said for RTS,S. Consequently, an adjuvant system is required to boost immunogenicity, some of the best results for which have been obtained using ASO1 and ASO2. These are proprietary formulations of GSK's AS class of adjuvant system. ASO1 uses liposomes mixed with immunostimulants 3-O-deacylated monophosphoryl lipid A and portion 21 (QS-21) of Quillaja saponaria, a plant native to South America. ${ }^{[54,56]}$ ASO2 uses water-inoil in place of liposomes but retains the same immunostimulants. These adjuvants boost antibody and $T$ cell responses, thus escalating cell-mediated immunity. ${ }^{[57]} \mathrm{A}$ specific benefit is their capacity to stimulate Th1 rather than Th2 $\mathrm{CD}^{+}{ }^{+}$cells. ${ }^{[54]}$ This is advantageous because the Th1 subset is the only one to produce IFN- $\gamma$.

The immune system has the ability to remove sporozoites from the blood and to prevent development within hepatocytes; however, it is not totally effective. It is not fully understood why only partial immunity to malaria is induced but it has been theorised that the shedding of CSP-antibody complexes may act to avoid detection. ${ }^{[58]}$ If RTS,S is successful, a way around this problem may be achieved.

\section{RTS,S/AS01 PHASE 3 CLINICAL TRIALS}

Of over 20 vaccine projects that are in clinical trials in 2014, phase 3 clinical testing of RTS,S/ASO1 is at least 5-10 years ahead of other candidate vaccines. ${ }^{[36]}$ This is being conducted in over 15,000 infants and young children in seven sub-Saharan African countries: Burkina Faso, Gabon, Ghana, Kenya, Malawi, Mozambique and Tanzania. ${ }^{[59]} \mathrm{A}$ range of locations was selected in order to enable evaluation of the vaccine's effectiveness under different conditions of malaria transmission. Of the two age groups in the trial, infants received three doses of RTS,S/ASO1 together with other routine childhood vaccines at 6,10 and 14 weeks of age. Older children were aged between 5-17 months at first dose of the vaccine.

\section{Early indicators of efficacy of RTS,S/AS01 vaccine protection}

The first results from the phase 3 trial were published in October 2011 for children aged 5-17 months at first immunization. ${ }^{[60]}$ The estimated overall efficacy was a $55 \%$ reduction in all clinical episodes of $P$. falciparum infection during the 12 months of follow-up, with $47 \%$ efficacy against severe, life-threatening cases estimated in this age group. Data for children vaccinated at 6-14 weeks of age, in co-administration with other vaccines, were released in November 2012. ${ }^{[61]}$ Estimated overall efficacy in this age group over 12 months of follow-up was $33 \%$ for all malaria episodes, and $37 \%$ for severe infection.

There is evidence in both age groups that protection declines over the year following vaccination, and it is not known yet how long protection extends beyond this period. Furthermore, whether a booster dose is required to enhance protection remains to be established. The implications of the apparent difference in level of protection afforded by RTS,S according to age of the recipient include the need for a thorough assessment of the feasibility, safety and effectiveness of different possible schedules and immunization strategies for this vaccine.No data are available yet to indicateif the level of protection varies between field sites with different

Cite as: Taylor-Robinson AW. Progress on The Path to A Licensed Vaccine Against Plasmodium falciparum Malaria. J Immunol Immunotech. 2014:1 (1)-1-14. 
intensities of malaria transmission. More information on all of these issues should be available soon after the completion of the phase 3 trial later this year.

A detailed analysis is required to explore reasons for the apparent lower efficacy when RTS,S is administered to infants rather than to older children. Possible causative factors include interference by other co-administered vaccines, maternally acquired antibodies, transmission intensity and seasonality. An initial finding is that lower immune responses are induced by the vaccine in infants aged 6-14 weeks compared to children aged 5-17 months.

\section{Predicted availability of RTS,S/AS01 vaccine for African children}

If the full results of the current phase 3 trial of RTS,S provide sufficient evidence of a protective effect against $P$. falciparum, arguably it could be considered a 'first generation' malaria vaccine. ${ }^{[62,63]}$ This means that RTS,S would be recognised as partially effective, reducing the number of cases of malaria in vaccinated children, but not preventing all episodes of the disease. Before recommendation for introduction into immunization programs in endemic countries, information is needed on how long the vaccine's protection lasts, the efficacy of a booster dose and what the protection level is in different settings in Africa.

Any such vaccine would require licensure by national regulatory authorities, evaluation of which becomes relevant when sufficient safety, immunogenicity and efficacy data for the target population for immunization is available. A number of requirements would then need to be fulfilled. ${ }^{[62]}$ These include: a WHO recommendation for use in a designated target population; WHO prequalification for a specific vaccine formulation from a specific manufacturer to ensure international standards of quality, safety and efficacy are met (for countries wishing to be supplied through the United Nations, or which use WHO prequalification as the basis for procurement eligibility); and implementation by national public health agencies of an agreed program of vaccine delivery. The role a novel intervention is projected to playin the context of existing preventive and treatment measures together with its affordability and cost-effectiveness are just two examples of the many additional factors beyond efficacy that will influence a country's decision-making on introduction. Based on what is now known, and depending on the final trial results, a WHO recommendation for use and subsequent prequalification of RTS,S may occur as early as 2015 .

\section{Conclusions}

The need for an effective, affordable vaccine against $P$. falciparum malaria mortality and morbidity and the associated negative socioeconomic impacts is a global public health imperative. Proof that a vaccine is feasible is based on two facts. First, knowledge that repetitive exposure leads to naturally acquired non-sterile immunity, and second that sterile immunity can be induced using subunits, and indeed attenuated sporozoites. With this in mind and considering the mass of supportive data, a malaria vaccine seems attainable within the next decade. This would be in accord with the Malaria Vaccine Technology Roadmap, published in 2006, through a collaboration of the world's foremost health organizations including the WHO, MVI, BAMGF and the Wellcome Trust. ${ }^{[23]}$ This unveiled a highly ambitious target to attain a vaccine by 2025 that demonstrates protective efficacy of over $80 \%$ against clinical disease caused by P. falciparum and lasts for over four years. A key measurable outcome is that by 2015 the vaccine should be $50 \%$ effective against severe disease and death and should last for a minimum of 12 months.

Although RTS,S could be the world's first licensed malaria vaccine, its performance in trials has not proved as strong as hoped or indeed required. Trials have yet to show that RTS,S can induce herd immunity which is ultimately necessary to meet the NGOs' goal of malaria eradication. Moreover, while it may induce sterile

Cite as: Taylor-Robinson AW. Progress on The Path to A Licensed Vaccine Against Plasmodium falciparum Malaria. J Immunol Immunotech. 2014:1 (1)-1-14. 
immunity, as with naturally acquired immunity, this is imperfect and in trial subjects this eventually waned. The full results of the phase 3 trial will go a long way to determine whether or not RTS,S has a viable future, at least in its present formulation. If deemed to be unsuccessful - and, since the results may not be clear cut, this will require consensus - other candidate vaccines will probably become the focus of attention. This begs the question of what is the minimum acceptable efficacy in trials for a vaccine to continue to commercialisation since even a modestly effective malaria vaccine would protect hundreds of thousands of people from disease and death each year. Perceived failure would reinforce the contention of some protagonists in the malaria research community that vaccine development is not best use of resources and investment in existing control measures should be the directive. Putting diversion of funds to one side, these different intervention strategies complement each other so a pragmatic policymaker would be wise to adopt a multifaceted approach with a vaccine as just one of the tools used to reduce the global burden of $P$. falciparum.

\section{ACKNOWLEDGEMENTS}

The author's research receives support from the Australian Commonwealth Health Collaborative Research Network and Central Queensland University.

\section{REFERENCES}

1. Moya A, Font E. Evolution: From Molecules to Ecosystems. UK, Oxford: Oxford University Press; 2004.

2. Singh B, Kim Sung L, Matusop A, et al. A large focus of naturally acquired Plasmodium knowlesi infections in human beings. Lancet. 2004;363(9414):1017-1024.

3. World Health Organization. World Malaria Report 2008. Geneva: WHO; 2008.

4. Breman JG. Eradicating malaria. Sci Prog. 2009;92(1):1-38.

5. U.S. Census Bureau. International Programs - World POP Clock Projection. 2011. Retrieved from: http://www.census.gov/ipc/www/ popclockworld.html

6. Hay SI, Guerra C, Tatem A, et al. The global distribution and population at risk of malaria: past, present, and future. Lancet Infect Dis. 2004;4(6):327-336.

7. Guerra CA, Gikandi PW, Tatem AJ, et al. The limits and intensity of Plasmodium falciparum transmission: implications for malaria control and elimination worldwide. PLoS Medicine. 2008;5(2):e38.

8. World Health Organization. Malaria. 2010. Retrieved from: http://www.who.int/mediacentre/factsheets/fs094/en/

9. Malaria Vaccine Initiative. The PATH Malaria Vaccine Initiative. 2010. Retrieved from: http://www.malariavaccines.org/publications-factsheets.php

10. Malaria Vaccine Initiative. Accelerating Progress Toward Malaria Vaccines. 2007 Retrieved from: http:// www.malariavaccines.org/files/080212_MVI_portfolio_bro_mvilogo_000.pdf

11. Sherman IW. Malaria - Parasite Biology, Pathogenesis and Protection. Washington DC: ASM Press; 1998.

12. Gallup JL, Sachs JD. The economic burden of malaria. Am J Trop Med Hyg. 2001;64(1-2):85-96.

13. Targett GAT, Greenwood BM. Malaria vaccines and their potential role in the elimination of malaria.

Cite as: Taylor-Robinson AW. Progress on The Path to A Licensed Vaccine Against Plasmodium falciparum Malaria. J Immunol Immunotech. $2014: 1$ (1)-1-14. 
Malaria Journal. 2008;7(S1):S10.

14. Markell EK, Voge M, John DT. Medical Parasitology, 7th edition. London: W.B. Saunders; 1992.

15. Centers for Diseases Control. DPDx - Malaria. 2011. Retrieved from: http://www.dpd.cdc.gov/DPDx!HTML/malaria.htm

16. Knell AJ. Malaria:a publication of the tropical programme of the Wellcome Trust. Oxford: Oxford University Press; 1991.

17. Bruce-Chwatt LJ. Bruce-Chwatt's Essential Malariology, 4th edition. London: Hodder Arnold; 2002.

18. Mueller I, Galinski MR, Baird JK, et al. Key gaps in the knowledge of Plasmodium vivax, a neglected human malaria parasite. Lancet Infectious Diseases. 2009;9(9):555-566.

19. Greenwood BM. Control to elimination: implications for malaria research. Trends in Parasitology. 2008;24(10):449-454.

20. Slutsker L, Kachur S.P. It is time to rethink tactics in the fight against malaria. Malaria Journal. 2013;12:140.

21. Stevenson MM, Zavala F. Immunology of malaria infections. Parasite Immunol. 2006;28(1-2):1-4.

22. Narasimhan V, Attaran A. Roll Back Malaria? The scarcity of international aid for malaria control. Malaria Journal. 2003;2(8):1-8.

23. Roadmap Working Group. Malaria Vaccine Technology Roadmap. 2006. Retrieved from: http://www. malariavaccineroadmap.net

24. Kurtis JD, Mtalib R, Onyango FK. Human resistance to Plasmodium falciparum increases during puberty and is predicted by dehydroepiandrosterone sulfate levels. Infection and Immunity. 2001;69(1):123128.

25. Baird JK, Jones TR, Danudirgo EW, et al. Age-dependent acquired protection against Plasmodium falciparum in people having two year exposure to hyperendemic malaria. Am J Trop Med Hyg. 1991;45(1):65-76.

26. Baird JK. Age-dependent characteristics of protection v. susceptibility to Plasmodium falciparum. Ann Trop Med Parasitol. 1998;92(4):367-390.

27. Smith TA, Leuenberger R, Lengeler C. Child mortality and malaria transmission intensity in Africa. Trends Parasitol. 2001;17(3):145-149.

28. Taylor-Robinson AW. Validity of modelling cerebral malaria in mice: argument and counter argument. Journal of Neuroparasitology. 2010;1:1-5.

29. Kane EG, Taylor-Robinson A.W. Prospects and pitfalls of pregnancy-associated malaria vaccination based on the natural immune response to Plasmodium falciparum VAR2CSA-expressing parasites. Malar Res Treat. 2011;2011:764845.

30. Taylor-Robinson AW, Morley LC, Kane EG. Rationale for pregnancy-associated malaria vaccination predicated on antibody-mediated immunity to Plasmodium falciparum placenta-binding parasites. In: Vaccines: Benefits and Risks. iConcept Press, Sunnybank Hills, QLD, Australia, pp. 95-130, 2013.

31. Abdel-Wahab A, Abdel-Muhsin AM, Ali E, et al. Dynamics of gametocytes among Plasmodium falciparum

Cite as: Taylor-Robinson AW. Progress on The Path to A Licensed Vaccine Against Plasmodium falciparum Malaria. J Immunol Immunotech. 2014:1 (1)-1-14. 
clones in natural infections in an area of highly seasonal transmission. J Infect Dis. 2002;185(12):18381842 .

32. Schofield L, Mueller I. Clinical immunity to malaria. Current Molecular Medicine. 2006;6(2):205-221.

33. Taylor-Robinson AW. A model of development of acquired immunity to malaria in humans living under endemic conditions. Medical Hypotheses. 2002;58(2):148-156.

34. Malaria Vaccine Initiative. Clinical Trials: Crucial Steps on the Road to a Malaria Vaccine. 2004. Retrieved from: http://www malaria vaccine.org/files/M VI_clinical_trials_paper.pdf

35. Morley LC, Taylor-Robinson AW. Understanding how Plasmodium falciparum binds to the placenta and produces pathology provides a rationale for pregnancy-associated malaria vaccine development. Open Vaccine Journal 2012;5:8-27.

36. Denny JWL, Taylor-Robinson AW. The development pathway of the most advanced candidate vaccine against malaria, RTS,S. Journal of Malaria Research 2012; 2(1):1-29.

37. Malaria Vaccine Initiative. Fighting malaria today and tomorrow. 2008. Retrieved from: http://www. malariavaccines.org/publications-factsheets.php

38. World Health Organization. World Malaria Report 2009. Geneva: WHO; 2009. Retrieved from: http:// www.who.int/malaria/world_malaria_report_2009/en/

39. Hill AVS. Pre-erythrocytic malaria vaccines: towards greater efficacy. Nature Reviews Immunology. 2006;6(1):21-32.

40. Mikolajczak SA, Aly ASI, Kappe SHI. Pre-erythrocytic malaria vaccine development. Current Opinion in Infectious Diseases. 2007;20(5):461-466.

41. Malaria Vaccine Initiative. Fact Sheet: RTS,S Malaria Vaccine Clinical Trials. 2006. Retrieved from: http://www.malariavaccine.org/files/FS_RTSS_FINAL.pdf

42. Ballou WR, Arevalo-Herrera M, Carucci D, et al. Update on the clinical development of candidate malaria vaccines. Am J Trop Med Hyg. 2004;71(S2):S239-247.

43. Ballou WR, Cahill CP. Two decades of commitment to malaria vaccine development: GlaxoSmithKline biologicals. Am J Trop Med Hyg. 2007;77(6):289-295.

44. Stoute JA, Kester KE, Krzych U, et al. Long-term efficacy and immune responses following immunization with the RTS,S malaria vaccine. J Infect Dis. 1998;178(4):1139-1144.

45. Waitumbi JN, Anyona SB, Hunja CW, et al. Impact of RTS,S/ASO2(A) and RTS,S/ASO1(B) on genotypes of $P$. falciparum in adults participating in a malaria vaccine clinical trial. PLoS One. 2009;4(11):e7849.

46. Ambroise-Thomas P. Vaccination against malaria. Disappointments and hopes. Bull Acad Natl Med. 1997;181(8);1637-1648.

47. Doolan DL, Martinez-Alier N. Immune response to pre-erythrocytic stages of malaria parasites. Curr Mol Med. 2006;6(2):169-185.

48. Plebanski M, Hill AVS. The immunology of malaria infection. Curr Opin Immunol. 2000;12 (4):437-441. 49. Perlmann P, Troye-Blomberg M. Malaria Immunology, 2nd edition. Basel: Karger; 2002.

Cite as: Taylor-Robinson AW. Progress on The Path to A Licensed Vaccine Against Plasmodium falciparum Malaria. J Immunol Immunotech. $2014: 1$ (1)-1-14. 
50. Reece WH, Pinder M, Gothard PK, et al. A CD4 ${ }^{+} \mathrm{T}$-cell immune response to a conserved epitope in the circurmsporozoite protein correlates with protection from natural Plasmodium falciparum infection and disease. Nature Medicine 2004;10(4):406-410.

51. Cruse JM, Lewis RE. Atlas of Immunology, 2nd edition. London: CRC Press; 2004.

52. Malaria Vaccine Initiative. Sanaria PfSPZ malaria vaccine candidate. 2009. Retrieved from: http:// www.malariavaccines.org/ publications-factsheets.php

53. Ballou WR, Arevalo-Herrera M, Carucci D, et al. Update on the clinical development of candidate malaria vaccines. Am J Trop Med Hyg. 2004;71(S2):S239-247.

54. Richie T. High road, low road? Choices and challenges on the pathway to a malaria vaccine. Parasitology. 2006;133(suppl.):S113-144.

55. Sun $P$, Schwenk R, White $K$, et al. Protective immunity induced with malaria vaccine, RTS, S, is linked to Plasmodium falciparum circumsporozoite protein specific $\mathrm{CD}^{+}$and $\mathrm{CD} 8^{+} \mathrm{T}$ cells producing IFN- $\gamma$. Journal of Immunology. 2003;171(12):6961-6967.

56. World Health Organization. WHO - Parasitic Diseases. 2011. Retrieved from: http://www.who.int/vaccine_research/diseases/soa_parasitic/en/index4.html\#pre-erythrocytic\%2Ovaccines

57. Antigenics Inc. QS-21 immune adjuvant. 2010. Retrieved from: http://www.antigenics.com/products/ tech/qs2

58. Stewart MJ, Vanderberg JP. Malaria sporozoites leave behind trails of circumsporozoite protein during gliding motility. J Protozool. 1988;35(3):389-393.

59. Leach A, Vekemans J, Lievens M, et al. Design of a phase III multicenter trial to evaluate the efficacy of the RTS,S / ASO1 malaria vaccine in children across diverse transmission settings in Africa. Malar J. 2011;10:224.

60. Agnandji ST, Lell B, Soulanoudjingar SS, et al. RTS,S Clinical Trials Partnership. First results of phase 3 trial of RTS,S/ASO1 malaria vaccine in African children. N Eng J. 2011;365(20):1863-1875.

61. RTS,S Clinical Trials Partnership, Agnandji ST, Lell B, et al. A phase 3 trial of RTS,S/ASO1 malaria vaccine in African infants. N Eng J. 2012;367(24):2284-2295.

62. Birkett AJ, Moorthy VS, Loucq C, et al. Malaria vaccine R\&D in the decade of vaccines: breakthroughs, challenges and opportunities. Vaccine. 2013;31(suppl. 2):B233-243.

63. Denny JWL, Taylor-Robinson AW. Progress with RTS,S - the most advanced candidate vaccine against malaria. In: Peterson A.M.,Calamandrei G.E.(eds.), Malaria: Etiology, Pathogenesis and Treatments. Nova Science Publishers, Hauppauge, NY, USA, pp. 39-67, 2012.

Cite as: Taylor-Robinson AW. Progress on The Path to A Licensed Vaccine Against Plasmodium falciparum Malaria. J Immunol Immunotech. $2014: 1$ (1)-1-14. 